\title{
La malédiction : pouvoir de faibles ou redoutable pouvoir?
}

The Curse: Power of Weaks or Redoutable Power?

\section{Samia Khichane}

\section{(2) OpenEdition}

1 Journals

Édition électronique

URL : https://journals.openedition.org/clo/5884

DOI : $10.4000 /$ clo.5884

ISSN : 2266-1816

Éditeur

INALCO

Édition imprimée

Date de publication : 18 juin 2019

Pagination : 81-117

ISBN : 978-2-85837-348-8

ISSN : 0396-891X

\section{Référence électronique}

Samia Khichane, "La malédiction : pouvoir de faibles ou redoutable pouvoir ? », Cahiers de littérature orale [En ligne], 85 | 2019, mis en ligne le 19 juin 2020, consulté le 22 mars 2022. URL : http:// journals.openedition.org/clo/5884; DOI : https://doi.org/10.4000/clo.5884

\section{(c) (i) (9)}

Cahiers de littérature orale est mis à disposition selon les termes de la Licence Creative Commons Attribution - Pas d'Utilisation Commerciale 4.0 International. 


\title{
La malédiction : pouvoir de faibles ou redoutable pouvoir?
}

\author{
Samia Khichane \\ LACITO (CNRS/Sorbonne nouvelle/Inalco)
}

De nombreux parlers contemporains possèdent un répertoire de formules de malédictions qui occupe une place de choix dans les situations de conflits. Dans le monde musulman, les travaux menés sur ce sujet proviennent de documents hagiographiques, traductions et chroniques, mais aussi d'enquêtes de terrain au sein de sociétés actuelles (Amri-Salameh, 2006 ; Gokalp, 2004 ; Taine-Cheikh, 2004) qui mettent en avant la malédiction en situation de conflits. Citons par exemple l'article de Nelly Amri-Salameh sur la malédiction Du'â' des saints de l'Ifrîqiyya médiévale qu'elle définit comme « un puissant levier de l'action du saint, de son intervention, et l'un des fondements de son pouvoir » (Amri-Salameh, 2006, p. 69) tant pour celui qui sollicite la médiation du saint que lorsque celui-ci est personnellement pris à partie. Dans la langue turque contemporaine, la malédiction [ilenti] est selon Altan Gokalp d'un usage commun chez les Turcs, mais elle est aussi une forme de pouvoir, parce qu'elle s'apparente aux genres littéraires les plus savants comme l'épopée. Dans ce contexte, l'usage de l'invective et de la malédiction est « d'une certaine manière une prétention à la distinction et à la domination de l'autre » (Gokalp, 2004). À l'inverse, dans le contexte judéo-arabe marocain, la pratique de la malédiction est d'après Norman Arthur Stillman une compensation de cette minorité tributaire et assujettie à la domination de la majorité non-juive. 
Les membres des abl al-dimma ${ }^{1}$ n'avaient la permission ni de porter des armes, ni de frapper un musulman [...]. Mais les invectives et les malédictions - tout comme l'humour - sont l'arme des faibles. (Stillman, 2008, p. 13)

Dans les différentes études qui sont présentées, il apparait que la pratique de la malédiction reflète des rapports de domination qui varient selon les cultures, mais aucune ne semble faire mention d'un usage socialement genré de la malédiction. L'enquête de terrain sur laquelle repose cet article a été effectuée dans une zone essentiellement kabylophone au sud-ouest de la wilaya ${ }^{2}$ de Tizi-Ouzou (Algérie). Elle s'appuie sur une approche ethnographique des conflits domestiques (Khichane, 2018) à partir d'un corpus recueilli au sein des trois communautés $(\mathrm{A}, \mathrm{B}, \mathrm{C})$ issues de mon lignage maternel ${ }^{3}$. Cette étude ethnographique révèle qu'en Kabylie, la malédiction est surtout une pratique langagière répandue chez les femmes. Parce que les femmes sont socialement dominées, nous verrons que le recours à des pratiques détournées telle que la malédiction est pour elle l'unique moyen de se défendre des représailles que leur inflige la société.

\section{Parole efficace et mots interdits : un détour par l'euphémisme}

Dans la société traditionnelle kabyle, le contrôle de la langue par le groupe et l'usage qu'en fait l'individu est très fort. L'euphémisme est particulièrement bien représenté au niveau lexical de manière omniprésente, ainsi que le précise Salem Chaker (1997), de sorte que la nomination d'un certain nombre de réalités est soumise à des contraintes très strictes, que ce soit dans le domaine privé ou public, en présence du sexe opposé ou de ses aînés (Ibid.). Il souligne que :

Tout ce qui relève (ou simplement peut évoquer) la sexualité et la reproduction, les fonctions corporelles, la mort, le sort (mauvais),

1. Ce terme désigne dans le droit musulman des citoyens non musulmans liés à un État musulman par un pacte de protection.

2. Division territoriale de l'Algérie qui équivaut à département en France.

3. L'étude d'une approche ethnographique des conflits domestiques est en soi une entreprise très délicate. Elle l'est d'autant plus dans une société traditionnelle à domination masculine encore très soucieuse de son honneur, en particulier lorsqu'elle porte atteinte à la femme. Le choix de mener mon enquête au sein d'un terrain de familiarité s'est donc imposé pour recueillir des données (Khichane, 2019). 
certains animaux (de mauvais augure),... s'il ne peut être évité, doit être dénommé de manière indirecte. (Ibid., 1997, p. 2706)

La foi en la puissance du verbe génère également des interdits langagiers, notamment en tout début de journée. Les transgresser équivaut à prononcer de redoutables incantations, car porteurs de mauvais présages, ils pourraient avoir un effet néfaste. Comme Pierre Bourdieu le faisait déjà remarquer :

De tous les mots interdits, les plus redoutables, au matin, sont tous ceux qui expriment des actes ou des moments terminaux, fermer, éteindre, couper ou, à moindre degré, finir, épuiser, partir, répandre, propres à évoquer une interruption, une destruction avant terme, le vide ou la stérilité. La foi dans le pouvoir des mots implique que l'on mette des formes dans les rapports avec le monde, toute transgression, verbale ou gestuelle, des formes prescrites pouvant avoir des effets cosmiques. (Bourdieu, 1980, p. 419)

Lorsqu'une réalité ne peut être évitée, il est nécessaire de la contourner par la dénomination indirecte. L'euphémisme servira alors à rompre l'effet malfaisant des mots et devient une contrepartie de la malédiction. On dira par exemple : « buferreh » [celui qui réjouit] pour désigner l'« adajin » (qui est un plat à cuire les galettes) parce que la suie relevée du dessous de ce plat servait autrefois à marquer le deuil, enduite sur le visage. Cet ustensile est de ce fait un présage de mort. L'euphémisme (« celui qui réjouit ») permet d'inverser le présage funeste qu'il symbolise. De même, la chouette, oiseau malfaisant qui représente la mort, est appelée « amerbuh » qui signifie « le béni » ou bien alors « le bénéfique ». Lorsque les lamentations de cet oiseau nocturne se font entendre, il est conseillé de bénir ses petits afin que ceux des hommes soient épargnés par le malheur :

Dieu, protège ses petits et protège les nôtres.

A Rebbi hrez arraw-is hrez arraw-nney.

VOC Dieu IMP2S- protéger. AOR enfants=POSS3S IMP2S-protéger.

AOR enfants $=$ POSS1P

Pour une requête qu'on ne peut satisfaire, il est de bon augure de répondre :

Il n'y a que du bien

D lxirkan

PP bien seulement 
plutôt que : «ulac » [il n'y a rien], sous peine d'appeler la ruine sur soi et sur les siens. À celui qui formule une demande en commençant sa phrase par ulac, on dira :

Puisse Dieu te réduire à néant.

A k-iger Rebbi d ulac.

POT DIR2MS=SUJ3MS- faire. AOR Dieu PP NEG.EX

L'enfant demandera du pain à sa mère en disant : «yella weyrum ? » [y a-t-il du pain ?] plutôt que : « ulac ayrum ? » [il n'y a plus de pain ?]. Il se gardera également d'exprimer sa faim en termes de : «lluẓey » [j'ai faim], mais par la périphrase : « byiy ad ččey » [j'ai envie de manger]. Autrement, l'enfant est blâmé pour son manque de discernement « d yir lfal » [c'est un mauvais présage] et repris afin qu'il acquière la bonne manière de parler : « mačči akka i qqaren medden ${ }^{4} \gg$ [les gens ne parlent pas de la sorte]. Cette attitude apparaît comme un fait d'ordre culturel qui est renforcé par l'apprentissage de la langue et des stratégies discursives. Elle rentre dans une éducation morale qui selon Laouste-Chantréaux (1990) témoigne de plus de sévérité encore envers la fille qu'envers le garçon. D'une part, parce qu'elle se doit d'éviter de prononcer certains mots qui peuvent être inconvenants mais surtout parce que la parole féminine est plus redoutée, à l'instar des malédictions.

\section{Maudire par le mal-dire}

La malédiction est désignée en kabyle par l'expression ddeewa n ccerr composée de deux termes d'origine arabe : daca [appeler, demander, invoquer] et ccerr [méchanceté, mal, malheur], qui signifient littéralement « invocation du mal » (mauvais sort, malheur, conséquence du mal qu'on fait), par opposition à la bénédiction qui se dit ddeswa $n$ lxir traduite par «invocation du bien ». Il est nécessaire de ne pas confondre avec ddeعwessu, composé des mots ddeعwa et ssu, qui signifie « malédiction, punition, malheur, conséquence du mal qu'on fait » (Dallet, 1982, p. 169). Il s'agit d'un châtiment résultant de toute atteinte portée sur tout ce qui est considéré comme sacré : leznaya [la protection donnée], les esprits tutélaires, les parents ddeewessu n lwaldin (litt. « malédiction des parents ») (Abrous, 2010). Cette notion serait l'équivalent en français de « malédiction » définie dans le Dictionnaire de langue française comme : «Malheur auquel on

\footnotetext{
4. mačcilakka/i/qqaren/medden

NEG.ATT/comme cela/RELI/SUJ3MP- dire. AORI/gens.
} 
semble voué par la destinée, par le sort » (Rey et al, 2014, p. 1515). Autrement dit, les deux acceptions de «malédiction » sont rendues en kabyle par des locutions différentes. La première acception, ddeewa n ccerr, est l'acte de langage par lequel on invoque une puissance transcendante pour qu'elle frappe autrui. La deuxième acception, ddecwessu, désigne une situation d'infortune, en général causée par une puissance transcendante, mais sans qu'aucun acte de langage particulier en ait été la cause.

L'islam offre également en héritage le terme de lesna qui signifie « écarter loin, priver du bien » et dont seul Dieu a la légitimité. Quand Dieu maudit une personne - pour avoir commis un acte à cause duquel il a mérité la malédiction comme la consommation du vin, du fruit de l'usure ou l'insulte de ses parents ou d'autres grands péchés - Il la prive de Sa miséricorde et la rend passible d'un châtiment, d'où sa perte. Proférée par les hommes, elle signifie « injurier et invoquer Dieu contre quelqu'un ». La malédiction consiste en une délégation qui est faite à Dieu d'une sanction qui ne peut être donnée par soi-même. L'invocation devient dans ce cas un acte perverti qui n'est pas admis par l'islam.

La forme canonique de la malédiction se présente comme un vœu, une invocation qui est faite à Dieu. Elle peut être interpellative et s'adresser directement à une personne (énonciateur/énonciataire) en face à face. Exemple :

Que Dieu te brûle !

Ak-yezzu Rebbi!

POT DIR2MS=SUJ3MS-brûler. AOR Dieu

Que Dieu t'enlaidisse !

Ak-yecemmet Rebbi!

POT DIR2MS=SUJ3MS-enlaidir. AOR Dieu

La malédiction peut être référentielle et s'adresser à un « adressataire » (celui à qui s'adresse l'énonciateur) à propos d'une personne absente (destinataire). Exemple :

Que Dieu l'anéantisse !

A t-iqucc Rebbi!

POT DIR3MS=SUJ3MS-anéantir. AOR Dieu

La malédiction peut également s'adresser matériellement à un adressataire à propos d'un destinataire (absent), mais avec un énoncé dont la structure grammaticale s'adresse directement au destinataire. C'est le cas de cette belle-mère qui, remontée contre sa bru jugée indocile, se plaignait à son mari des méfaits de 
cette dernière. Soudain, tout en s'adressant à son époux, elle leva les mains vers le ciel et dit (à sa bru absente) :

Puisse Dieu t'arracher de ma maison même avec la tête de mon fils ! A kem-yeqles Rebbi seg wexxam-iw ulukan s uqerruy $n$ mmi.

POT DIR2FS=SUJ3MS-arracher. AOR Dieu de maison. EA=POSS1S si avec tête. EA de mon fils

C'est également le cas de cette femme qui me relatait les méfaits de sa belle-sœur et l'invectiva soudainement, en ma seule présence mais s'adressant explicitement à l'absente :

Comme mon mari m'a battue, le tien te battra ; ce que tu as regardé en moi, je le regarderai en toi. (Sens caché : comme tu as joui de mes malheurs, je jouirai des tiens).

Akken yewwet țtbel fell-i ad yewwet țtbel fell-am, $i$ tferğed deg-i ad ferğey deg-m.

Comme SUJ3MS-frapper. PRET tambour sur=PRP1S POT SUJ3MS-frapper.AOR tambour sur=PRP2FS RELI SUJ1S-regarder. PRET dans=PRP1S POT SUJ1S-regarder.AOR dans=PRP2FS

La cible d'une malédiction peut riposter en retournant sur son énonciateur le mauvais présage.

Soit par exemple qu'une personne dirige sur une autre le premier mot ou la première partie de la formule de malédiction et que la cible riposte en complétant l'énoncé. L'énonciateur dira alors :

Que Dieu te donne la mort !

Am-d-yefk Rebbi lmut!

POT IND2FS=PROX=SUJ3MS-donner. AOR Dieu mort.

Ce à quoi répondra l'énonciataire devenant énonciateur à son tour :

Qui t'emportera.

A kem-yeddmen.

POT DIR2S=SUJ3FS-emporter. AOR

Un deuxième exemple de malédiction formulée selon ce même procédé : Nacera secoue le tapis de son salon par la fenêtre. Sa voisine du dessous, assise sur son balcon, reçoit toute la poussière sur sa tête et se met alors à crier. Nacera qui n'avait pas de mauvaises intentions, aurait préféré qu'elle l'aborde plus amicalement et lui dit : 
Que Dieu mange ta tête!

Ad yečc Rebbi aqerruy-im!

POT SUJ3MS-manger. AOR Dieu tête=POSS2FS

Ce à quoi sa voisine riposta :

Qu'il rajoute ta tête et celles de tes enfants !

Ad yernu aqerruyim d uqerruy $n$ warraw-im!

POT SUJ3MS-rajouter. AOR tête=POSS2FS et tête. EA de enfants.

$\mathrm{EA}=\mathrm{POSS} 2 \mathrm{FS}$

Le verbe « manger » est employé par la première femme avec l'intention d'appeler à la disparition de sa voisine. Pour triompher sur sa rivale, la seconde femme inclut la descendance de Nacera, en appelant ainsi à l'anéantissement de sa lignée.

Soit par le recours à des formules telles que :

Puisse Dieu faire que la malédiction se réalise en toi !

Ad iger Rebbi d-gem!

POT SUJ3MS- faire. AOR Dieu dans-PRP2FS

ou bien encore « af uqerruy-im ${ }^{5}$ » [sur ta tête !] comme dans l'exemple de cette belle-mère qui ne s'accommodait guère des visites fréquentes que sa bru faisait à ses parents, lesquels habitaient à un pâté de maisons. Un jour, alors que sa bru s'apprêtait à se rendre chez ses parents, elle lui fit part de son désaccord, mais la jeune femme ne le prit pas en considération. À peine avait-elle mis le pied dehors que la vieille femme l'invectiva :

Que Dieu te plante un clou au pied.

A m-d-yefk Rebbi amesmar deg udar.

POT IND2FS=PROX=SUJ3MS-donner. AOR Dieu clou dans pied. EA

Ce à quoi répliqua la belle-fille :

Sur ta tête! (Puisse ta malédiction retomber sur ta tête).

Af uqerruy-im.

Sur tête. EA=POSS2FS

Puis s'en alla voir ses parents en toute sérénité.

5. af / uqerruy-im

Sur / tête. EA=POSS2FS 


\section{Le serment ou la malédiction conditionnelle}

Le serment se dit en kabyle agalli [prêter serment, jurer] comme dans l'expression : « Yeggul ur yehnit! » [Il a juré et n'a pas manqué à son serment ${ }^{6} !$ ] (Haddadou, 2014, p. 201), mais aussi lemsahda [serment, promesse solennelle] (Ibid., p. 748). Ce terme vient du verbe arabe wasada qui signifie « prêter serment ». La forme canonique du serment se présente selon la typologie d'Évelyne Larguèche (1998) comme «quelqu'un [qui] jure par/sur/devant quelqu' un ou quelque chose ». En kabyle, le verbe employé dans l'énoncé du serment est en général ggal [jurer] par exemple :

Je jure par Dieu que...

Gulley s Rebbi ar...

SUJ1S-jurer. PRET par Dieu que

Je jure par le serment de Dieu que...

Gulley s lemsacda Rebbi ar...

SUJ1S-jurer. PRET par serment Dieu que

ou bien promettre solennellement [ $[$ ahed $]$.

Je fais le serment par Dieu que...

Eusdey s Rebbi ar...

SUJ1S-faire le serment. PRÊT par Dieu que

On emploie également l'expression a heqq [par le nom de].

Par le nom de Dieu.

A heqq Rebbi.

par le nom de Dieu

Par le nom du Cheikh Ben Abderrahmane.

A heqq ccix Ben Abderrahmane.

par le nom de cheikh Benabderrahmane

Ce type de serment entretient avec la malédiction une relation étroite de sorte que les deux modalités peuvent se confondre, comme dans l'exemple de cette femme dont la belle-sœur s'était moquée en raison des multiples césariennes qu'elle avait subies. Elle proféra contre elle la malédiction suivante : 
Tu t'es moquée de moi pour mes opérations, alors je jure par Dieu que toi aussi tu en subiras.

Gulley ar ad tt-txedmed ula d kemmini.

SUJ1S-jurer. PRET que POT DIR3FS=SUJ2FS=faire. AOR même PP toi

Dans cet exemple, la malédiction prend la forme d'un serment du type « Je jure par Dieu qu'il t'arrivera tel malheur », mais il s'agit là d'une malédiction qui n'a que trompeusement l'apparence d'un serment. Peut-on prêter serment à propos de quelque chose dont on n'est pas maître et qui ne peut venir que de Dieu ? Dire « je jure par Dieu que toi aussi tu [...] subiras [des opérations] », ce n'est pas prêter serment, mais user d'une manière particulière de dire « Que Dieu fasse qu'il t'arrive tel malheur ».

Un second type de serment peut s'identifier à la malédiction, celui qui se présente sous la forme d'une proposition conditionnelle introduite par un terme à valeur hypothétique : «ma » $\mathrm{si}]$. On parle dans ce cas de « malédiction conditionnelle »(Zagnoli, 1991). Ferroudja accuse la sœur de son mari de lui avoir subtilisé une robe qu'elle avait mise à sécher. Cette dernière s'en défend en ces termes :

Que Dieu ruine notre maison si j'ai volé ta robe.

Ad yexlu Rebbi axxam-nney ma yella ukrey taqendurt-im.

POT SUJ3MS-ruiner. AOR Dieu maison=POSS1P si SUJ3MS-être.

PRET SUJ1S-voler. PRET robe=POSS2FS

Pour Giorgio Agamben (2009) tous les serments se concluent nécessairement sur une malédiction dans la mesure où, en cas de parjure, l'individu s'expose à la colère de la divinité invoquée qui se transforme en vengeur et qui lui fait subir le châtiment énoncé sous la forme optative. D’un point de vue linguistique, le destinataire de la malédiction n'est pas, dans ce cas, le destinataire de l'énonciation. Le serment apparait, ainsi que le résume bien Nello Zagnoli (1991), comme la rencontre entre une promesse ou une assertion et une automalédiction.

Soit, mais si l'on prend l'exemple ci-dessus, dire : «Que Dieu ruine notre maison si j'ai volé ta robe » n'est qu'une manière particulièrement forte de dire : « Je jure devant Dieu que je n'ai pas volé ta robe » et l'automalédiction qui s'y ajoute ne fait que donner plus de force à ce serment. La personne qui jure ne souhaite évidemment pas que sa maison soit ruinée. Le fait qu'elle donne à son serment la forme d'une malédiction ne fait qu'attester qu'elle dit vrai car, à moins d'être inconsciente, elle ne prendrait pas le risque de se mettre ainsi à la merci de la colère divine. 
Nous avons donc dans un cas une malédiction qui prend l'apparence d'un serment, mais ce n'est qu'une apparence. Dans l'autre cas, nous avons un serment qui s'adjoint une malédiction pour se donner plus de force. La malédiction est bien présente, mais elle n'est là qu'au service du serment. Et comme la personne qui prête serment lui a donné une forme conditionnelle, elle sait (si elle dit vrai) qu'elle ne risque rien.

La forme canonique du serment féminin se distingue fondamentalement du serment masculin dans le choix du garant ou du témoin. Toutefois, cette opposition est neutralisée lorsque le garant est Dieu ou un saint puisque les hommes comme les femmes y ont recours sans contrevenir aux codes de comportements langagiers. La femme jure sur la tête de quelqu'un avec une tendance à l'ellipse du verbe. Dans la plupart des cas, le garant est un ascendant ou un descendant masculin direct de la femme (le père, le fils), plus rarement sa fille, si la femme n'a pas de fils ou de frère :

Sur la tête de mon fils.

$S$ uqerruy/ixf $n$ mmi.

Par tête. EA de mon fils

Sur la tête de mon père.

$S$ uqerruy $n$ baba.

Par tête. EA de mon père

Sur la tête de mes enfants.

$S$ uqerruy $n$ warraw-iw.

Par tête. EA de enfants. EA=POSS1S

Dans une imprécation du genre « je jure sur la tête de mon fils », le rôle que joue le fils de celle qui jure est différent de celui que joue Dieu, ou un saint dans un serment comme « je jure devant Dieu » ou d'un serment avec malédiction conditionnelle comme « que Dieu ruine notre maison si j'ai volé ». Dieu est implicitement présent dans la formule « je jure sur la tête de mon fils » car Lui seul sévira en cas de mensonge. Il serait plus judicieux d'appeler « caution », ou « gage », le rôle joué par le fils dans ces formules, plutôt que « témoin » ou « garant ». La caution est une garantie qu'on perd en cas de manquement à un engagement, exactement comme le fils dans le cas d'un parjure.

Les femmes emploient également l'expression « je vendrais la tête de mes enfants » comme forme de serment qui sert à se disculper face à une accusation. Fatma a eu huit filles. À l'âge de quarante ans, elle donne naissance à un garçon qui décède au bout de quelques heures en raison d'une malformation au niveau de la colonne vertébrale. Quelques années plus tard l'une de ses filles qui a deux petites 
filles et la quarantaine passée décide de faire un autre enfant avec l'espoir que ce soit un garçon. L'une de ses sœurs la traite alors de folle [taderwict] en lui expliquant qu'elle risque d'avoir un garçon sans colonne vertébrale comme leur mère, fait qu'elle a appris de leur tante maternelle. Intriguée, la fille de Fatma va voir sa mère et lui fait part de ce qu'elle vient d'apprendre, laquelle se hâte de prendre son téléphone afin de s'expliquer avec sa sœur. Pour se défendre de ce dont on l'accuse, cette dernière fait le serment à sa sœur qu'elle serait prête à vendre la tête de ses enfants pour lui prouver qu'elle n'a jamais dit mot de cette histoire à qui que ce soit :

Je vendrais la tête de mes enfants.

Ad zzenzey aqerruy $n$ warraw-iw.

POT SUJ1S-vendre. AOR tête de enfants. EA=POSS1S

Très souvent, le serment est pour une femme une manière de s'imposer une auto-sanction en cas de parjure. L'expiation d'un jeûne serait, par exemple, le quelque chose par/sur/devant lequel une femme s'engage pour donner du poids à sa parole. Fatiha invita les beaux-parents de son fils à déjeuner et se donna beaucoup de peine à préparer des mets délicieux en leur honneur. Une fois la table dressée, la belle-mère du jeune homme ne daigna pas prendre une bouchée de nourriture, pas même boire une gorgée d'eau ce qui est la moindre des politesses, puisque ce serait une manière de suggérer à ses hôtes que l'on ne ressent pas de dégoût à leur égard ou que l'on ne craint pas d'eux un acte de sorcellerie. Fatiha insista en lui proposant une tasse de thé ou de café, mais elle refusa en expliquant qu'elle était rassasiée. Vexée par l'attitude de son invitée, elle lui fit le serment de ne plus manger chez elle si elle s'obstinait à refuser de prendre quoi que ce soit :

Que la kafara ${ }^{7}$ me soit imposé si je mange à nouveau chez toi.

Telzem-iyi tkeffart ma cawdey ad čcey deg wexxam-im.

SUJ3FS-imposer. PRET =IND1S jeûne expiatoire.EA siSUJ1S-recommencer.

PRET POT SUJ1S-manger. AOR dans maison. EA=POSS2FS.

Ou l'exemple de cette mère qui poursuivait son enfant afin de le réprimander :

Kafara de soixante jours si je ne te frappe.

Takeffart $n$ settin yum $m$ ur txelsed.

Jeûne expiatoire de soixante jours si NEG SUJ2S-payer. AOR

7. Promesse d'un sacrifice expiatoire d'un serment par le jeûne. 
L'auto-sanction consiste pour un homme à s'interdire sa propre femme ${ }^{8}$. « Que ma femme me soit illicite si tu t' introduis sur mon terrain » dit un homme à son voisin qui lui avait demandé de lui céder quelques mètres de son terrain pour y faire passer des buses d'assainissement.

Que ma femme me soit illicite.

\section{Tehrem tmettut-iw.}

SUJ3FS-interdire. PRÊT femme. EA=POSS1S

Le même serment émis par une femme, à propos de son époux, provoque des réactions différentes : elle est passible de répudiation. L'assemblée du village $\mathrm{A}$ intervint pour résoudre un litige qui opposait un mari à sa femme afin de rétablir la paix conjugale sans que leur différend ne s'étalât dans les tribunaux. Lors de cette réunion étaient présents : les membres de l'assemblée du village, le mari ainsi que les frères de l'épouse (en tant que porte-parole de cette dernière). La parole fut donnée en premier lieu au mari qui expliqua qu'il ne tolérait plus les sorties incessantes de sa femme ainsi que les allées et venues des voisines. Ces comportements lui avaient valu une mauvaise réputation auprès du voisinage, celle d'être un « homme sans autorité ». Il imposa comme condition que son épouse lui obéisse davantage à l'avenir et que les sorties ne se fassent plus sans lui, ou tout au moins avec son consentement. La parole fut ensuite donnée aux frères de l'épouse qui acquiescèrent, mais demandèrent, néanmoins, l'avis de la femme concernée qui rétorqua en ces termes : « Si telles sont tes conditions, alors je répudie tes moustaches si je remets les pieds chez toi. »

Je répudie tes moustaches.

Briy icclayem-ik.

SUJ1S-répudier. PRET RELI moustaches=POSS2MS.

Les membres de l'assemblée, scandalisés, quittèrent brusquement la réunion en déclarant qu'ils ne pouvaient assister à une scène où la femme répudiait son mari et, plus grave encore, ses moustaches.

\footnotetext{
8. Bourdieu (2000) explique ce comportement par le fait que l'épouse fait partie à la fois de ce qui est sacré et de ces choses honteuses qu'on ne nomme qu'en s'excusant, dans la mesure où l'honneur du mari et de toute sa famille s'érige autour d'elle. Il est toutefois nécessaire de préciser que l'épouse en tant que femme n'est pas à proprement parler une chose honteuse, elle est un être par qui la honte peut arriver (lorsqu'elle déshonore les siens par son inconduite). Elle rend les siens vulnérables à la honte, mais n'est pas honteuse en elle-même.
} 


\section{Une parole au rythme du quotidien}

Dans l'univers domestique, la malédiction est une pratique en usage avant tout chez les femmes, notamment dans le cadre des querelles et des disputes qui les opposent entre elles ou (très rarement) aux hommes. Elle intervient également dans leurs rapports à leurs enfants, comme pratique éducative.

\section{Avec les enfants}

La forme la plus simple de la malédiction est celle adressée aux enfants. Elle se présente littéralement comme un vœu simple qu'une maladie, voire un malheur s'abatte sur l'enfant. Les malédictions sont proférées si souvent à l'égard des enfants qu'il n'est pas nécessaire de les prononcer dans leur totalité, mais seulement sous une forme abrégée. Elles se présentent comme des énoncés à un seul terme, comme par exemple : lfula [une/la carie], tiwermin [une/l'angine], rrheğ [un/le poison], ou encore à deux termes composés d'un substantif et de son qualifiant zzaweq amsewweq [un/le mercure déroutant], ssem asemmam [un/le poison acide], tawla tașemmat [une/la fièvre froide] ou d'un substantif suivi d'un complément :

Poison en poudre des poules.

Ssahuq n iyuzad.

poison en poudre des poules. EA

Galle des chameaux.

Ajeğğid n iluyman.

Galle des chameaux

Dans les malédictions adressées aux enfants, le nom de Dieu peut également être formulé dans l'imprécation. Ces malédictions sont énoncées par des mères à l'égard d'enfants trop turbulents. Celles qui portent sur les maladies sont parmi les plus utilisées : les images que véhiculent ces malédictions renvoient pour la plupart à des maladies qui touchent très souvent les enfants (la fièvre, la galle, la rage de dent). L'invocation dans ce cas renvoie à l'idée de douleur, mais surtout à l'effet qu'elle a sur l'enfant alité et inapte à faire des bêtises. Très souvent, l'imprécation est suivie de l'énumération des effets escomptés par la mère :

Puisse Dieu te donner une rage de dent qu'elle te fasse entortiller dans ton lit !

Ak-d-yefk Rebbi tuymest a k-tesseymumes. POT IND2MS=PROX=SUJ3MS-donner. AOR Dieu rage de dent POT DIR2MS=SUJ3FS-faire entortiller. AOR 
Puisse Dieu te donner une fièvre qu'elle te calme !

A k-d-yefk Rebbi tawla a k-tessers.

POT IND2MS=PROX=SUJ3MS-donner. AOR Dieu Fièvre POT

DIR2MS=SUJ3FS-calmer. AOR

Puisse Dieu te donner une angine pour que tu te taises !

A k-d-yefk Rebbi tiwarmin meqqar ad tessusmed.

POT IND2MS=PROX=SUJ3MS-donner. AOR Dieu angine en moins

POT SUJ2S- se taire. AOR

Les désastres font également partie du répertoire d'invectives adressées aux enfants :

Que Dieu te donne la foudre, qu'elle te prenne!

Ak-d-yefk Rebbi ssieqa, a k-teddem!

POT IND2MS=PROX=SUJ3MS-donner. AOR Dieu foudre POT

DIR2MS=SUJ3FS-emporter. AOR.

Puisse Dieu te donner un torrent, qu'il t'emporte !

A k-d-yefk Rebbi tahemmalt a k-tawi!

POT IND2MS=PROX=SUJ3MS-donner. AOR Dieu torrent POT

DIR2MS=-SUJ3FS-prendre. AOR

Le souhait dans ce cas précis ne renvoie pas à un présage funeste, mais plutôt à celui d'écarter momentanément de sa vue l'enfant turbulent. La mort est très rarement invoquée à l'égard d'un enfant, ainsi que les maladies graves, mais lorsque c'est le cas, la mère neutralise juste après l'effet néfaste de ses paroles par des formules prophylactiques émises à voix haute ou chuchotées à l'oreille de l'enfant qu'elle console à l'instar de :

Mon Dieu protège mon enfant!

A Rebbi hrez-iyi mmi!

VOC Dieu IMP2S-protéger. AOR= IND1S mon fils

Les malédictions employées par les femmes kabyles dans leurs rapports avec les enfants consistent en un apprentissage social que fait l'enfant. Il s'agit donc par le recours à la malédiction de signaler l'interdit et de donner une « sanction fantasmatique » du geste répréhensible (Lallemand, 1975) qu'elle réalise par une « délégation langagière » (Miliani 1986). Ces constructions sont associées de manière plus ou moins arbitraire à un signifié bien précis. Leurs effets performatifs, entendus comme volonté de nuire à la personne, sont atténués et frappés de nullité par l'usage répété de ces malédictions. Chaque malédiction se rapporte à un 
contexte précis, redondant et ancré dans la mémoire. Dans la plupart des cas, il s'agit d'inculquer à l'enfant, par le biais de la malédiction, des règles de politesse et de lui signaler l'interdit. La réprimande prend la forme d'une malédiction qui vise à éliminer les comportements prohibés, dont on peut faire la liste à partir des exemples suivants :

\section{Lagloutonnerie}

La mère dit à son enfant qui mange trop ou qui subtilise la part de son frère : «rrheğ » [un/le poison].

Pour le déjeuner, la maman prépare des frites. Au fur et à mesure qu'elle sort une série de la friteuse, les enfants en prennent un peu dans une assiette jusqu'à ce qu'il n'en reste plus : «tafruxt ! » [un/l'oiseau femelle], leur dit-elle ; autrement dit : «Arrêtez de picorer comme des oiseaux ! »

\section{Les burlements}

$\grave{A}$ un enfant qui crie trop fort, on dit : « tuymest » (litt. « une/la dent ») qui signifie « rage de dent ».

Une maman enlève des ciseaux des mains de son fils de quatre ans; il crie tellement fort qu'elle lui dit : «lfula » [une/la carie !].

Les enfants jouent dans le couloir alors que leurs mères, occupées à la cuisine, discutent entre elles. Un moment les femmes ne s'entendent plus parler à cause des enfants qui jouent à qui crie le plus fort; la grand-mère furieuse leur lance : « tiwermin » [une/l'angine !].

Une maman prépare à manger dans la cuisine, l'aîné de ses enfants âgé de six ans veut regarder un dessin animé alors que sa sœur à peine plus jeune que lui veut en regarder un autre. Ils viennent se plaindre à leur mère qui, pour les punir, éteint la télévision. Le garçon furieux tire sur les cheveux de sa sœur, laquelle lui assène un coup sur le ventre ; le souffle coupé, il lâche les cheveux de sa sœur qui va se cacher sous les jupons de sa mère. Le garçon en larme fonce droit vers sa mère qui essaye de le calmer, puis le petit frère de deux ans se joint à la bande en donnant des coups de pieds à son frère et à sa sœur. La maman, furieuse, tire les deux grands par leurs vêtements en direction de leur chambre en leur criant dessus :

Puisse Dieu vous donner la foudre !

Ad awen-d-yefk Rebbi ssicqa!

POT IND2MP=PROX=SUJ3MS-donner. AOR Dieu foudre

Dans l'après-midi, pour se reposer après les différentes tâches ménagères effectuées dans la matinée, les femmes s'installent devant la télévision pour regarder leur feuilleton quotidien. L'une des filles de la maisonnée, âgée de douze ans, se 
met à commenter chaque action du film, la robe de l'actrice, les décors, tout et n'importe quoi, ce qui finit par agacer tout le monde et à embarrasser sa mère qui lui crie dessus : « Tais-toi ! Tu nous brûle nos têtes en parlant, tu as mangé une cassette aujourd'hui! », en poursuivant :

Que Dieu mange ta tête.

Ad yečc Rebbi aqerruy-im.

POT SUJ3MS-manger. AOR Dieu tête=POSS2FS

\section{Les rots et les flatulences}

À celui qui rote ou qui émet une flatulence, on dira : «bucehị̣ » [un/le choléra des poules].

Pendant que les femmes discutent entre elles, les enfants ont souvent l'habitude d'écouter les conversations passionnantes en se blottissant dans le giron de leur mère où en jouant entre eux à même le sol. Soudain, l'un d'entre eux laisse échapper un pet accompagné d'une odeur nauséabonde, ce qu'il lui vaut une malédiction : « bucehịạ » [un/le choléra des poules], « on dirait que tu as mangé un chien ».

Levol

Une maman donne à manger à son petit de deux ans, puis se lève de table un instant. L'aîné de ses enfants arrive et dérobe le dessert de son petit-frère ; celui-ci se met à pleurer. « Rrheğ » [un/le poison !] lui dit sa mère qui n’a pas pu le rattraper.

Pour la fête de l'Aïd, les femmes de la maison ont préparé des beignets qu'elles ont soigneusement cachés. Les enfants, ayant découvert la cachette, se sont tous servis à l'insu de leurs mamans. Pris sur le fait, les petits chenapans prennent la fuite alors que leur tante leur lance :

Puisse Dieu vous donner de la poudre à canon au ventre !

Ad iger Rebbi lbarud deg ucebbud-nwen.

POT SUJ3MS-donner. AOR Dieu poudre à canon dans ventre.

$\mathrm{EA}=\mathrm{POSS} 2 \mathrm{MP}$.

\section{Les grimaces}

Une maman regarde la télévision. Son petit de six ans lui fait des grimaces en retroussant le nez et en tirant la langue afin d'attirer son attention et la faire rire. Elle le regarde avec dédain puis lui met la main sur le visage de sorte à défaire sa grimace et dit : 
Que Dieu te couvre d'opprobre!

A k-yexzu Rebbi.

POT DIR2MS=SUJ3MS-couvrir d'opprobre. AOR Dieu

\section{Les comportements dangereux}

Une petite fille de deux ans joue sous la surveillance des femmes de la maison. La porte du balcon s'ouvre, elle s'échappe et tente d'escalader les barreaux. Sa tante arrive en courant et lui crie :

Que Dieu te plante tu vas tomber!

A kem-yezzu Rebbi.

POT DIR2FS=SUJ3MS-planter. AOR Dieu

\section{L'agitation}

L'après-midi, tous dans la maisonnée font une sieste sauf le petit garçon et sa sœur qui, au sommet de l'excitation, s'amusent à courir dans le couloir. Dans leur trajectoire, ils marchent sur la grand-mère endormie sur sa natte en peau de mouton. Réveillée en sursaut, elle leur lance :

Fièvre froide, qu'elle vous calme!

Tawla tasemmadt a ken-tessers!

Fièvre froide POT DIR2P=SUJ3FS-calmer. AOR

À l'heure de la sieste, le petit garçon de quatre ans a du mal à s'endormir avec sa mère. Il court partout, monte sur les meubles de la maison au grand malheur de sa grand-mère qui ne peut se reposer de peur qu'il ne se fasse mal. Elle le met en garde, mais aussi, prie sa mère de faire quelque chose pour l'endormir, sans résultat. La grand-mère finit par s'assoupir un moment ; c'est alors qu'il prend une chaise pour monter sur la table. Il s'agrippe avec un genou, puis s'accroche à la nappe. La nappe glisse et le garçon tombe à la renverse, faisant tomber la chaise avec lui. La grand-mère se lève en sursaut et crie sur la maman :

Que Dieu te brûle, ton fils est tombé !

A kem-yezzu Rebbi!

POT DIR2FS=SUJ3MS-brûler. AOR Dieu

La maman, furieuse accourt auprès de son enfant en criant trois fois de suite : 
Puisse Dieu te donner une entorse, puisse Dieu te donner une entorse, puisse Dieu te donner une entorse?

Taruzi ncallah.

Entorse si Dieu le veut

Après une fessée, elle le prend dans son lit puis il s'endort en larmes quelques instants plus tard.

Les malédictions adressées aux enfants témoignent également d'une réalité sociale, celle de la claustration des femmes. Ne pouvant poursuivre l'enfant à l'extérieur de la maison, la mère délègue à Dieu, par l'intermédiaire de la malédiction, la sanction qu'elle ne peut infliger elle-même à son enfant. Prenons par exemple cette mère qui demandait à son fils aîné de surveiller son petit-frère en sortant un peu jouer dehors avec lui. Le fils aîné promit à sa mère de garder son frère plus tard, mais cette dernière ne voulut rien entendre, car elle était occupée et avait besoin de lui immédiatement. À peine s'était-elle retournée que son fils avait déjà pris la fuite. Comme elle ne pouvait le rattraper, elle lui adressa l'invective suivante :

Puisse Dieu te donner un coup !

A k-d-yefk Rebbi tiyita.

POT IND2MS=PROX=SUJ3MS-donner. AOR Dieu coup

Le garçon se retourna avec un rire nerveux vers sa mère qui le menaça en tirant sur le menton et lui disant : « a-tt-a fell-ak » [ho, si je t'attrape !], puis se tapa sur les mains en les frottant mimant le geste de le frapper.

Il arrive parfois que les femmes, poussées à bout par leurs enfants, s'auto-invectivent comme dans les expressions :

Stérilité de cent ans!

Ticiqqert n myat sna.

Stérilité de cent ans.

Ô mon Dieu enlève moi ou prends-moi !

A Rebbikkes-iyi ney ddem-iyi.

VOC Dieu IMP2S=enlever. AOR=DIR1S ou IMP2S=prendre.

AOR=DIR1S

9. La répétition n'a pas de signification particulière. Dans cet exemple, la mère répète la malédiction trois fois, ce qui correspond au temps parcouru pour arriver jusqu'à son fils. 
Comme pour les précédentes, ces malédictions expriment un sentiment d'exaspération. Il s'agit d'une circonlocution qui signifie « je veux mourir » ou « je n'en peux plus » qu'on peut également observer dans les malédictions relevées par Stillman (2008) dans le contexte judéo-arabe marocain, notamment lorsqu'une mère invective son enfant à l'aide de l'expression (ô orphelin/ô orpheline) pour signifier en réalité « tu me tues ». Ces malédictions comme celles qui s'adressent aux enfants n'ont pas le caractère funeste que véhicule leur sens littéral qui est neutralisé par la récurrence de son emploi dans des contextes bien spécifiques, à l'inverse des malédictions échangées dans le cadre d'une vraie dispute, telles que nous allons les voir, avec intention de nuire.

\section{Entre femmes}

Les malédictions qui ont pour énonciataires des femmes sont introduites par les formules suivantes : « que Dieu te... », « puisse Dieu faire... » ou «va-t'en... » en début d'énoncé (ces formules peuvent également être employées dans les malédictions dirigées à l'égard des enfants). Ces formules fonctionnent comme des indices de canonicité contribuant à rendre la forme de la malédiction plus conforme à l'attente d'une norme culturelle. Les malédictions les plus fortes sont celles qui appellent à l'anéantissement de quelqu'un par la répudiation :

Que Dieu t'arrache (te déracine) d'ici (de cette maison)!

A kem-yeqles Rebbi sya!

POT DIR2FS=SUJ3MS-arracher. AOR Dieu d'ici

Par la mort de l'énonciataire :

Que Dieu te donne celui qui te fera disparaître!

A m-d-yefk Rebbi win a kem-yeksen.

POT IND2FS=PROX=SUJ3MS-donner. AOR Dieu celui POT

DIR2FS=SUJ3MS-enlever. AOR

Ou de la descendance de celui-ci :

Que Dieu coupe tes repousses!

Ad yegzem Rebbi ixulaf-im.

POT SUJ3MS-couper. AOR Dieu repousses=POSS2FS

L'énonciation d'une malédiction s'attaque rarement à un homme, mais cela peut arriver quand, par exemple, une vieille femme énervée dit à sa bru : 
Que Dieu t'arrache à cette maison même avec la tête de mon fils ! A kem-yeqles Rebbi seg wexxam-agi ulukan s uqerruy $n$ mmi! POT DIR2FS=SUJ3MS-arracher. AOR Dieu de maison. EA=DEM si avec tête. EA de mon fils

Les malédictions introduites par «va-t'en » expriment le souhait de couper les liens avec son adversaire. Ainsi, lors d'un conflit entre femmes, l'une des deux protagonistes menaça de quitter la maison sans jamais y remettre les pieds en ces termes : « Je vais laver mes pieds sur la porte. » La réponse à cette menace fut alors :

Va-t'en, puissions-nous ne plus nous revoir !

Ruh a wer timlilit.

IMP2FS-partir. AOR POT NEG rencontre

On emploie également l'expression :

Va-t'en, puisses-tu ne plus revenir !

Ruh a wer d-uyaled.

IMP2FS-partir. AOR POT NEG PROX=SUJ2FS-revenir. AOR

Ou encore :

Va-t'en, puisses-tu ne pas te retourner !

Ruh a werd-zzid.

IMP2FS-partir. AOR POT NEG PROX=SUJ2S-se retourner. AOR

Va-t'en, puisses-tu ne pas être heureuse.

Ruh a wer ssecd.

IMP2FS-partir. AOR POT NEG bonheur

Le pied qui t'emmène existe, celui qui te ramène, non.

Aḍar a kem-yawin yella Win a kem-id-yarren ulac.

Pied POT DIR2FS=SUJ3MS-prendre. AOR SUJ3MS-être. PRET celui POT DIR2FS $=$ PROX $=$ rendre. AOR NEG.EX

À l'instar du défunt qui est porté vers la tombe, il n'y a pas de personne - par métonymie de pied - qui puisse le ramener.

Les hommes sont très rarement visés par les malédictions du moins, comme on l'a vu précédemment, pas directement. Les situations observées sont quasiment inexistantes et les récits récoltés remontent pour la plupart aux expériences de la petite enfance, aux malédictions dont les hommes ont souvent été victimes, de la part de leur mère, de leur grand-mère, d'une vieille voisine ou d'une tante, et 
dont ils se souviennent encore. Les invectives étaient lancées en conséquence de leur agitation, voire de leur désobéissance. Au sein des différents foyers où j'ai élu résidence, les femmes prononcent très souvent des malédictions entre elles notamment dans le cadre de disputes ou à l'égard des enfants trop turbulents : filles et garçons. Passé l'âge de l'adolescence, les hommes cessent d'être les cibles des malédictions.

Parmi les exemples recueillis, les rares malédictions qui ont pour destinataires des hommes adultes ont eu lieu dans des situations où les deux protagonistes étaient physiquement séparés l'un de l'autre. Cette distance semble indispensable l'homme dans la rue, la femme sur le pas de la porte ou dans la maison - ainsi que l'atteste l'exemple suivant : alors que Mouloud et son ami passaient par un village voisin, ils s'introduisirent dans un champ pour manger quelques fruits et se ravitailler pour la route. La propriétaire, les voyant de loin, ne pouvait aller à leur poursuite ni même leur faire face. Impuissante, elle enchaîna, en criant à leur adresse, une série de malédictions :

Puisse Dieu vous donner un gros ver blanc!

A wen-d-yefk Rebbi qawsas!

POT IND2MP=PROX=SUJ3MS-donner. AOR Dieu gros ver blanc

Puisse Dieu vous donner une bombe de l'Allemagne qui vous

brûlera les intestins !

Awen-d-yefk Rebbi lkura n lalmany a wen-yeșsaryen izarman!

POT IND2MP=SUJ3FS-brûler. AOR intestins

Les deux compagnons effrayés par ces propos prirent la fuite sur le champ.

\section{Des agents porteurs de malédiction}

La malédiction est une parole dont on redoute l'efficacité, en particulier lorsqu'elle est émise par des agents détenteurs d'un pouvoir reçu légitimement qui rend leurs malédictions automatiquement opérantes. Parmi les malédictions qui retiennent mon attention, notamment dans le cas d'une énonciation féminine, celles qui sont lancées par les détenteurs de l'autorité parentale, par les veuves et par les agents liés par la commensalité et le partage du sel.

\section{Atteinte de l'autorité parentale : une malédiction en héritage}

Les personnes jouissant d'une autorité parentale ont le pouvoir d'accorder une bénédiction à leurs descendants. « Pour celui qui la reçoit, enfant, adolescent, adulte, c'est un trésor précieux, un réconfort, un motif de fierté » (Zerdoumi, 
1970, p. 178). À l'instar de Ferhat qui est aujourd'hui médecin et se rappelle sa grand-mère appelant sur lui une bénédiction lorsque plus jeune il lui administrait ses médicaments :

Que Dieu t'accorde un remède entre les mains (le pouvoir de guérir).

A k-yefk Rebbi ddwa deg ufus-ik.

POT IND2MS=SUJ3MS-donner. AOR Dieu remède dans main. $\mathrm{EA}=\mathrm{POSS} 2 \mathrm{MS}$

Les parents ont également le pouvoir de prononcer des malédictions sur la vie de leurs enfants. Ces malédictions, dit-on, poursuivent leur victime [ttabarent] et se transmettent d'une génération à l'autre jusqu'au Jugement dernier, elles sont comme dit l'adage :

Comme un clou planté dans du bois ${ }^{10}$

$D$ amesmar di lluh.

PP clou dans bois

La malédiction des parents [ddecwa $n$ lwaldin] est l'une des plus redoutée puisqu'elle est dotée d'un régime d'efficacité même lorsqu'elle n'est pas prononcée. Dans ce cas précis, la malédiction est une sanction résultant de toute atteinte portant sur les parents ou toute personnes détentrices de l'autorité parentale. Le châtiment est alors inéluctable, quand bien même le parent tourmenté pardonnerait la faute commise, comme l'exemple de ce jeune homme qui avait suscité la colère de sa mère lorsqu'elle découvrit qu'il se livrait à la fornication. Un jour qu'elle tentait de le dissuader de retomber dans le péché de chair, la discussion tourna à la dispute. Le jeune homme prit la fuite et, par défi envers sa mère, s'en alla rejoindre son amante. La mère, exaspérée par ce comportement, ne pouvait le suivre à l'extérieur ; elle demeura impuissante au pied de la porte. Mais, tout en le suivant du regard, elle lui lança cette invective :

Puisse Dieu te brûler comme tu m'as brûlé !

Ak-yessehreq Rebbi akken iyi-tessherqed!

POT DIR2MS=SUJ3MS-brûler. AOR Dieu comme DIR1S=SUJ2Sbrûler. PRET

Le soir même, alors que le jeune homme travaillait dans son garage, le moteur d'une voiture prit soudain feu et explosa. Ce dernier fut transporté à l'hôpital en

10.Zerdoumi (1970, p. 177) relève une autre variante de cet adage en kabyle : « La malédiction des parents est comme un clou dans une outre. » 
urgence pour brûlure aggravée. La mère regretta alors de s'être ainsi emportée, car elle se sentait coupable d'avoir émis cette malédiction, dans la mesure où, selon les croyances populaires, la malédiction proférée par un parent tourmenté est toujours exaucée. À partir de ce jour, le jeune homme cessa de voir son amante et prit la résolution de devenir pieux en se tournant vers la prière.

Dans de nombreuses sociétés, les femmes ont le pouvoir d'invoquer le sein maternel qui s'avère être d'une redoutable efficacité. Chez les Aït Khebbach, tribu du sud-est marocain : «le lait, dit-on, est plus fort que le sang [agho ichqa ugar idamn] » (Gélard, 2004, p. 59). Cette expression témoigne selon Marie-Luce Gélard de l'importance de la filiation par le lait, tout comme le fait de « jurer sur le lait [adach galch s-ogho] » (Ibid.), pratique invoquée dans les circonstances les plus solennelles. Dans ce contexte, la puissance du lait est telle qu'en cas de parjure : «si Dieu est susceptible de pardonner, le lait demeure quant à lui intraitable et les exemples donnés d'individus morts brutalement à la suite d'un tel parjure sont fréquents » (Ibid.). En pays Ngambay au Sud du Tchad, une des malédictions les plus redoutées est celle prononcée par une mère offensée en tenant son sein droit dans la main (Fédry, 2010). La filiation par le lait occupe également une place importante dans la culture italienne, notamment en Calabre où la mère a le pouvoir de maudire sa descendance en se dénudant le sein dans un acte symbolique de retirer le lait qui est désigné par «le serment du lait » (Zagnoli, 1991). En Kabylie, il semblerait que ce type de malédiction ait perdu de son efficacité puisqu'elle relève à présent du tabou. Citons l'exemple de Slimane qui avait recruté son frère cadet, Ahmed, comme vendeur dans son magasin à la demande de sa mère. Au bout de quelques mois, Slimane alla voir cette dernière pour l'avertir qu'il comptait renvoyer Ahmed à cause des vols à répétition dont il s'était rendu coupable. La mère tenta de raisonner Slimane en le priant de penser à ses neveux avant tout, mais en vain. Afin de le dissuader, elle fit le serment de ne pas lui pardonner s'il songeait à exécuter sa menace :

Je jure par le sein maternel qui t'a élevé que je ne te le pardonnerai pas.

$A$ heqq tabbuct-aki $i k$-id-yessekren ur $k$-semher.

Par le sein=DEM RELI DIR2MS=PROX=SUJ3FS-élever. PRET NEG

IND2MS=SUJ1S-pardonner. PRETN

Embarrassé par ces propos, le fils s'en alla sans dire mot. Quant aux femmes de la maisonnée qui avaient assisté à la scène, elles trouvèrent honteux, (« d leib » [c'est la honte]) de s'adresser de la sorte à son fils. De même pour cette autre mère que son fils accusa de favoriser son frère auquel elle avait remis à plusieurs reprises une partie de sa pension. Celle-ci s'en défendit en ramenant la main de son fils sur son sein : 
Au nom de ceci (en désignant son sein) que je ne fais pas de différence entre vous deux.

A heqq taki ur xedmey lxilaf ger-awen.

Au nom de celle-ci NEG SUJ1S-faire. PRETN différence entre=PRP2MP

Alors que le geste de ramener la main de son fils sur son sein était censé accorder à son serment plus de pouvoir et décupler la force de sa parole, il mit le fils dans l'embarras et coupa court à la conversation. Quant à la vieille femme, elle fut sermonnée par sa belle-fille pour son geste jugé inconvenant.

\section{La malédiction de la veuve chaste}

Les jeunes veuves ont également le pouvoir de prononcer des malédictions en invoquant leurs épreuves surmontées. En voici une situation dont j'ai moi-même été témoin durant mon enquête et qui met en scène Sadia, une vieille femme de 83 ans qui vit auprès de son fils et de ses petits-enfants. Bien qu'entourée et bien choyée, son âge avancé la rend très susceptible, de telle sorte que le moindre geste ou comportement venant de son entourage est souvent perçu par elle comme une provocation. Le mari de Sadia l'a laissée précocement veuve durant la guerre de libération alors qu'elle n'avait que 22 ans. Elle a dû faire face à toutes les misères du monde pour élever ses trois enfants. Il y a quelques années, sa fille unique est décédée à la suite d'un cancer. Son souvenir la hante, en particulier à la nuit tombante, raison pour laquelle elle préfère s'endormir entourée de ses quatre petites-filles. Lorsque ces dernières étaient encore en bas âge, cette situation ne posait pas de problème, mais à présent qu'elles sont grandes, les soirées se font plus longues à cause d'un examen prévu pour le lendemain, d'un feuilleton à la télévision ou d'une discussion avec des amis sur le net, etc. et l'heure du coucher des jeunes filles ne s'accorde plus avec celle de leur grand-mère.

Lorsque le muezzin annonce la dernière prière du soir [leica], aux alentours de neuf heures, Sadia appelle ses petites-filles pour aller se coucher, mais celles-ci préfèrent poursuivre leurs activités ou regarder leur série hebdomadaire. Elle se remet dans son lit, puis se relève quelque temps plus tard et la scène se renouvelle. La grand-mère prend alors son fils à témoin afin qu'il ordonne aux jeunes filles de se coucher. Celui-ci n'en fait rien, mais tente de la raisonner en lui expliquant que ses petites-filles ne veulent pas la contrarier, mais seulement regarder un film à la télévision. La grand-mère qui est à présent dans tous ses états, se précipite dans la chambre, tire son matelas de son lit et le traîne jusqu'au salon. Consterné, son fils ordonne alors à l'une de ses filles de remettre le matelas de sa grand-mère en place et de se coucher avec elle, puis il rejoint lui-même sa propre chambre. Loin de se satisfaire d'une seule de ses petites-filles, la grand-mère reprend de plus belle et 
interpelle une nouvelle fois les jeunes filles en leur demandant ce qu'elle a bien pu leur faire pour qu'elle soit isolée ainsi :

Vous ai-je enfanté une fille?

Scif-akwent-id taqrurt?

SUJ1S-avoir. PRET =IND2FP=PROX fille

Vous ai-je enfanté une bâtarde?

Sciv-akwent-id takluct?

SUJ1S-avoir. PRET=IND2FP=PROX bâtarde

Ces expressions rendent compte de manière implicite de deux idées. La première, celle d'un acte déshonorant, la seconde l'exclusion d'une personne en conséquence de cet acte dont elle se serait rendue coupable ${ }^{11}$. Sur ce, la bru prie la grand-mère de se calmer et de laisser les filles finir leur film, mais la vieille femme, au comble de sa colère, lance une série d'invectives à la bru ainsi qu'à ses petites-filles :

Puisse Dieu vous trahir !

A kwent-yexdec Rebbi!

POT DIR2FP=SUJ3MS-trahir. AOR Dieu

Puisse Dieu vous couvrir d'opprobre !

A kwent-yexzu Rebbi!

POT DIR2FP=SUJ3MS- couvrir d'opprobre. AOR Dieu

J'en appelle à ce que j'ai subi pour vous punir !

Wekkeley fell-akwen lectab-iw!

SUJ1S-s'en remettre. PRET sur-PRP2FP peine $=$ POSS1S

11. Mettre au monde une fille est source d'ennui pour sa famille d'une part, parce que l'honneur de son clan s'érige autour de sa pureté sexuelle, mais aussi parce qu'elle sera toute sa vie une source de dépense sans bénéfice, puisque ses parents peineront à l'élever pour qu'elle soit au service de sa belle-famille et continueront à lui pourvoyer des biens matériels après son mariage afin de hausser son prestige. Il arrive alors que la mère soit fâchée par sa belle-famille à la naissance d'une petite fille et autrefois elle pouvait même être répudiée si elle n'arrivait pas à donner naissance à un garçon. J'ai personnellement recueilli des témoignages de femmes qui avaient séjournaient plusieurs jours à la maternité, sans aucune visite de leur mari ni de leur belle-famille parce qu'elles avaient donné naissance à une petite fille. Le plus grave est la naissance d'une bâtarde qui souille l'honneur du lignage qui ne peut être lavé que par le sang versé de(s) coupable(s) ou par le reniement de la mère. 
En éloignant ses filles du salon, la mère riposte simultanément à chacune des malédictions par la réplique suivante : «Sur ta tête !» [uqerruy-im ${ }^{12}$ !]. Puis elle dit : « Alors, puisque c'est ainsi je jure par Dieu qu'elles ne dormiront pas avec toi ce soir ! »

Une veuve qui demeure célibataire par choix ou par contrainte - car le remariage est vécu comme un affront à la mémoire du mari défunt en particulier par sa belle-famille, ou par ses enfants - a également le pouvoir de maudire au nom de sa chasteté ${ }^{13}$ représentée par le port du foulard qui est la marque de « la soumission, de la honte et du mépris » (Yacine, 1995, p. 50), mais également par le bas de la robe qui est :

Comme une espèce de registre où sont consignées les actions des femmes. Les femmes qui ont été chastes (qui se sont privées d'amour) sont considérées comme des femmes qui ont le pouvoir de faire du mal si on les agresse. (Ibid., p. 51)

Les femmes veuves qui ont été chastes jurent par : «le foulard des jeunes veuves » (Yacine, 1995, p. 50), ou par « les épreuves d'une jeune veuve » (Ibid. p. 51) comme l'exemple de cette grand-mère citée plus haut. Elles ont également le pouvoir d'attirer la malédiction sur un offenseur en se libérant de leur foulard ou de leur ceinture. Elles sont alors dans la transgression (Ibid.) et c'est dans cette forme que j'ai pu recueillir un récit illustrant ce type de malédiction. Lorsque Hedjila perdit son mari dans les années soixante-dix, elle se retrouva veuve à l'âge de quarante-cinq ans avec trois jeunes enfants et un aîné qui était déjà marié. Ce dernier vivait dans la soupente aménagée de la maison [tacrict]. Fonctionnaire dans l'administration, il subvenait largement aux besoins de sa femme et de ses deux enfants en bas âge alors que ses parents vivaient à l'époque pour ainsi dire dans la misère. Quelques mois après la mort du vieux père, le fils aîné obtint une promotion et décida de quitter la maison pour s'installer à la capitale, dans un appartement plus spacieux, mais pas assez pour accueillir sa mère et ses frères. Le jour du départ venu, désemparée, sa mère tenta de le dissuader en le mettant en garde contre le péché $[d d n u b]$ qu'il commettrait en l'abandonnant à son sort, ce qui déclencha une violente dispute entre eux deux. D’un pas décidé, la belle-fille

\section{2. uqerruy-im}

Tête. EA=POSS2FS

13. En pays Ngambay au sud du Tchad, la chasteté est invoquée par la première épouse pour maudire son propre mari en cas de négligence ou de répudiation. La malédiction est alors introduite en ses termes : «c'est toi qui a brisé mon hymen » (Fédry, 2010, p. 53). 
s'interposa en poussant son mari à l'extérieur de la maison et en le rassurant en ces termes :

Sortons d'ici, il n'y a pas de péché.

Ffer ulac ddnub.

IMP2S-sortir. AOR NEG.EX péché

Elle se retourna vers sa belle-mère puis, en désignant du bout de son menton une dalle en ciment, elle lui dit :

Puisse Dieu faire que tu demeures ici comme cette dalle!

A kem-iger Rebbi am teylat-aki!

POT DIR2FS=SUJ3MS- faire. AOR Dieu comme dalle. EA=DEM.

La mère effondrée se précipita vers le cimetière du village pour se lamenter sur la tombe de son défunt mari puis, en se dégageant de son foulard, elle se mit à prononcer une succession de malédictions à l'encontre de son fils et sa belle-fille :

Je vous confie à Dieu pour vous punir.

Wekkeley-awen Rebbi.

SUJ1S-s'en remettre. PRET=IND2MP Dieu

Puisse Dieu faire que comme vous m'avez fait on vous fera.

Ad iger Rebbi akken iyi-txedmem a awen-yettwaxdem.

POT SUJ3MS-faire. AOR Dieu comme IND1S=SUJ2MP-faire. PRÊT

POT IND2MP=SUJ3MS-faire. AOR

Des gens du village l'avaient aperçue et accoururent pour la raisonner et la prier de revenir sur ses paroles qui risquaient de causer le malheur du fils et de son épouse, mais ceux-ci avaient à peine dépassé la périphérie du village qu'ils eurent un accident de voiture, duquel ils sortirent heureusement indemnes.

Le temps passa, et la vie de Hedjila prit un autre tournant, puisqu'elle vit à présent dans l'aisance auprès de l'un de ses fils cadets. À l'occasion de la fête de l'Aïd, alors que je séjournais pour mon enquête auprès d'elle, son fils aîné, accompagné de sa femme, vint lui rendre visite comme il a coutume de le faire à chaque fête religieuse. La vieille femme le questionna sur ses petits-enfants. Il lui confia que son épouse et lui n'avaient plus de nouvelles de leur fils qui avait pris ses affaires et quitté la maison quelques jours après son mariage. Elle lui dit alors :

Certains paient ici-bas, d'autres dans l'au-delà.

Kra ttxellișen di ddunit wyad di laxxert.

Certains SUJ3MP-payer. AORI dans monde les autres dans l'au-delà 
Le fils comprit par cette insinuation qu'il était en train de revivre ce que lui-même avait fait endurer à sa propre mère, sans compter les nombreux événements malheureux qui avaient traversé la vie de ce dernier : dépression nerveuse de sa femme, divorce de deux de ses enfants, etc., que sa mère et beaucoup de personnes de son entourage et aussi dans le village m'ont cité comme preuves de l'efficacité de la malédiction de la vieille femme. Mais celui-ci ne s'était jamais résigné à demander le pardon de sa mère. Hedjila s'est éteinte en décembre 2014. Sur son lit de mort, son fils cadet l'a suppliée de pardonner à son fils aîné, mais elle a refusé, puisque Dieu Lui-même ne pouvait lui pardonner.

\section{La commensalité, le sel et les liens sociaux}

La commensalité qui s'exprime métaphoriquement par le partage du sel est sacrée chez les Kabyles. C'est ainsi que les compères Ali et u-Ali se sont liés d'amitié, parce qu'ils ont peiné ensemble à se partager les aliments et le sel (Lacoste-Dujardin, 1970). Celui qui rompt «ce pacte symbolisant l'échange sacré du pain et du sel [tagela d melh] » (Yacine, 2001, p. 82) ne peut échapper à la malédiction qui lui sera adressée en conséquence puisque la sanction émanera du sel consommé :

C'est le sel consommé qui le frappera (C'est le sel consommé qui se chargera de le punir).

D lmelh-nni i yečca a t-yewweten.

PP sel=ANAPH RELI SUJ3MS-manger. PRÊT POT DIR3MS=SUJ3MS-frapper. AOR

Aussi il n'est pas nécessaire de prononcer une parole de malédiction pour que le briseur de lien soit puni :

Par Dieu je ne le maudirai pas, c'est le sel qu'il a consommé qui se chargera de le faire.

Wellah ma diziy-as d lmelh-nni is-cečcey a t-yewweten.

Par Dieu NEG SUJ1S-maudire. PRETN=IND3MS PP sel=ANAPH

RELI IND3MS=SUJ1S-faire manger. PRET POT DIR3MS=SUJ3MSfrapper. AOR

Le sel, en tant que symboles de liens se manifestant notamment par la commensalité, est doué d'une puissance immanente. En cela, il est le dépositaire de la valeur quasiment sacrée qu'on attribue à ces liens.

Les fruits du ciel comme de la terre, à l'instar du blé, de l'eau, du sel, etc., véhiculent un message culturel et une symbolique commune à tous les Kabyles. Pour déceler les différentes significations que revêt le sel dans cette société, il est nécessaire de l'analyser sur plusieurs niveaux. 
Le sel est utilisé comme condiment destiné à assaisonner des aliments ou des préparations culinaires mais aussi à la conservation des aliments tels que la viande. La salaison du mouton de l'Aïd [lexlie] permet de conserver la viande plusieurs mois, voire une année. Il est également lié aux mystères de l'univers que nous rencontrons dans un très grand nombre de croyances. En effet, le sel est le condiment le plus utilisé dans les pratiques magiques moyen-orientales, méditerranéennes et européennes, mais dans un rôle bien précis de repoussoir (Aubaile-Sallenave, 1988). Chez les Kabyles, le sel est utilisé contre les esprits malins, comme une défense frontalière qui protège le monde visible des humains du monde invisible des génies [djinns], voire une barrière protectrice à la lisière des lieux de passage entre le bien et le mal, la vie et la mort, la lumière et les ténèbres ${ }^{14}$. Pour se protéger de ces esprits, les femmes ont recours à des rites de protection. À chaque seuil qu'elles traversent et qui représente leur lieu d'habitation (pour prendre une douche, nettoyer les toilettes ou jeter de l'eau dans les gouttières par exemple), elles jettent du sel en prononçant une formule de recours à Dieu : «Que Dieu te maudisse et te confonde ô Satan ». Aussi, après un mauvais rêve, il est recommandé de jeter une poignée de sel sur le seuil de la porte de la maison afin d'empêcher que le présage véhiculé par le rêve ne vienne à se réaliser.

À la naissance, les mères posent dans le berceau du nouveau-né et jusqu’à ses quarante jours - qui, en contexte berbère, correspond d'après Marie-Luce Gélard (2016) à la phase transitoire durant laquelle la vie de l'enfant est particulièrement menacée - différents objets et aliments à valeur antimaléfique : un objet tranchant et un miroir afin d'effrayer les djinns (Ibid.), du goudron végétal, du sel, etc. (Aubaile-Sallenave, 1997). Lorsqu'un enfant est touché par le mauvais œil qui peut être la conséquence d'un regard insistant, d'une admiration sans volonté de nuire (tị $n$ lemhibba [œil de l'affection]), il est conseillé de prendre une poignée de sel dans sa main et de la tourner sur la tête de l'enfant sept fois du côté gauche et sept fois du côté droit.

Au quotidien, le sel signifie le repas partagé qui garde une grande place dans la vie et dans la culture kabyle « en tant que condiment [qui] ajoute un surcroit de valeur qui exprime les rapports solidaires des hommes entre eux » (Lacoste-Dujardin, 2003, p. 276). Plus qu'une nécessité vitale, il est l'une des formes de l'alliance par

14. Ainsi, selon des croyances religieuses, certains parmi les djinns vivent dans les endroits sales afin de se nourrir des restes jetés par les hommes, dans les cimetières et les ruines tandis que d'autres vivent parmi les hommes. On les trouve aussi dans les trous d'eaux sales et dans les égouts domestiques, raison pour laquelle ces esprits du mal sont tellement redoutés en particulier depuis la prière de l'après-midi [el $\varepsilon a s ̦ r]$ jusqu'à la nuit où ils règnent en maîtres. 
excellence et de la protection dans les populations du Moyen Orient et d'Afrique du Nord par le partage de nourriture (Aubaile-Sallenave 1988). Chez les Kabyles, partager un repas équivaut à créer un lien de solidarité, faire un pacte et sceller un contrat. Lors de la résolution des conflits ou litiges par les notables ou marabouts du village ou autres intermédiaires, la séance est clôturée par un repas rituel marquant la fin des hostilités comme gage d'inviolabilité des accords. Un repas qui rassemble les membres du groupe (protagonistes et intermédiaires) dans un acte à la fois de partage commun de nourriture et de réhabilitation de la solidarité sociale autour d'un plat en terre cuite [tabaqit] comme un engagement.

Les liens de solidarité sanctionnés par le partage du sel constituent une valeur à laquelle les Kabyles sont très attachés, d'où la mise en garde contre ceux qui s'aventureraient à les briser.

Dans la région des Aït Smail, la tradition veut que les proches parentes de la mariée, c'est-à-dire sa mère, ses grands-mères et ses tantes paternelles et maternelles qui l'accompagnent, restent avec elles jusqu'au lendemain du mariage appelé șṣbuh. Le lendemain du mariage de Kahina, alors qu'elle prenait le petit déjeuner avec ses parentes, sa belle-mère lui proposa de reprendre un peu de nourriture, mais pour des raisons de convenances, la jeune mariée refusa poliment. Autrefois, les femmes s'abstenaient de manger et de boire pendant une semaine, car il était inconvenant, voire honteux, pour une mariée d'avoir un besoin pressant d'uriner ou d'aller à la selle. La belle-mère insista puis dit à sa belle-fille, sur un ton plaisant :

Je vais te faire manger du sel (de la nourriture), de sorte que si je te maudis, je t'atteigne.

A m-cečcey lmelh akken ma yella ddciy-am a kem-hazey.

POT IND2FS=SUJ1S-faire manger. AOR sel pour si SUJ3MS-être. PRET

SUJ1S-maudire. PRET=IND2FS POT DIR2FS=SUJ1S-atteindre. AOR

Cela fit rire à contrecœur la mère ainsi que les tantes de la mariée, qui entendirent cette expression comme une menace. Selon elles en effet, loin de vouloir amuser les convives, la belle-mère avait plutôt l'intention de mettre en garde sa bru en exerçant son ascendant sur elle.

\section{Médiation et divinité}

La médiation par la divinité est l'une des stratégies auxquelles recourent les femmes pour se défendre, raison pour laquelle, selon Hadj Miliani (1986), on trouve dans les malédictions ce passage obligé, cette délégation faite à Dieu « punisseur ». La meilleure façon de punir un offenseur est de s'en remettre à Dieu. Parmi les ripostes utilisées, la menace d'une sanction divine : 
Taf, taf ${ }^{15}$, Dieu rattrape.

TTaf Țaf, Rebbi yettattaf.

Taf, taf, Dieu SUJ3MS-rattraper. AORI

L'évitement de la riposte est une forme de transcendance et de supériorité morale traduite par les valeurs communes. Néanmoins, la riposte est nécessaire afin de rétablir son image sociale et l'équilibre de l'honneur. Il apparait, selon mes observations de terrain, que le silence est une conséquence d'une injure qui marque sa victime du stigmate en lui ôtant toute possibilité de se reconstruire. Le stigmate fait partie de l'ensemble des réflexions de Goffman relatives aux rites d'interaction qu'il définit comme : « attribut qui jette un discrédit profond sur celui qui le porte » (Goffman, 2005, p. 13), de sorte que le sujet stigmatisé perd sa respectabilité et la possibilité de défendre sa face et de rétablir sa valeur sociale positive par le jeu rituel de l'interaction (Goffman, 1974). La malédiction intervient dans ce cas pour obtenir « justice », car la pratique de la malédiction résulte, ainsi que l'explique bien Atlan Gokalp :

[Du] désir de vengeance né du sentiment d'impuissance de celui qui réagit à l'iniquité vécue au tréfonds de son être, qui agit en son propre nom, par un énoncé et par la puissance de l'énoncé seulement. (Gokalp, 2004)

Le châtiment est alors imparable puisque la malédiction émise par un offensé tire son efficacité des tourments causés et subits. Pratiquement chaque année, on accueillait dans la maisonnée des B une naissance chez les deux belles-sœurs « tinudin ${ }^{16} »$, Zoubida, l'aînée, et Ouardia, la benjamine. Zoubida avait donné naissance, à l'époque de ce récit, à trois filles par voie naturelle et deux garçons non-viables pour lesquels elle avait dû subir des césariennes. Quelques semaines après la perte du second enfant, sa belle-sœur, Ouardia, a donné naissance à son troisième garçon.

Quelques mois plus tard, le petit garçon faisait sa première percée de dent. Selon la coutume, il est d'usage de préparer à cette occasion un plat de couscous avec de la viande. Comme les tâches ménagères étaient réparties entre les deux brus, ce jour-là, la préparation de ce plat incombait en principe à Zoubida. Elle s'apprêtait à le faire de bon cœur et demanda pour cela à la belle-mère de

15. Geste pressé de la main en écumant de colère.

16. Dans la terminologie de parenté kabyle le terme « tanuḍt » (plu. « tinuḍin ») désigne la femme du frère du mari. 
lui rapporter de la viande, mais cette dernière fit mine de n'avoir rien entendu. Zoubida se contenta alors de faire un couscous avec ce qu'elle avait sous la main. À l'exception de Zoubida et de ses filles, ni la belle-mère ni la bru, pas même les enfants de cette dernière n'ont daigné toucher au repas. On donna les restes au chien. Le lendemain, ce fut au tour de sa belle-sœur de s'occuper du déjeuner qui s'est déroulé, pour le coup dans une ambiance festive. La belle-mère chargea son fils d'acheter des poulets et des œufs et certaines voisines étaient même conviées pour midi, car il s'agissait officiellement du repas de la percée de dent du petit garçon.

Zoubida comprit alors que sa belle-mère et sa belle-sœur avaient mis de côté le repas préparé par ses soins de crainte qu'il ne porte malheur à l'enfant, étant donné qu'elle n'arrivait pas à maintenir des garçons en vie. Elle décida elle aussi de s'abstenir de manger en riposte à l'attitude que les deux autres femmes avaient eu la veille à son égard, ce qui lui causa de graves préjudices. Sa belle-sœur l'accusa de ne pas partager la joie de la famille par jalousie, puis s'acharna sur elle en prenant les convives à témoin : « regardez-la, la jalouse, un jour de joie et elle sème la discorde », puis, s'adressant à Zoubida elle dit :

C'est à cause de tes méfaits qu' Il (Dieu) te les donne (les garçons) et te les reprend.

$S$ lfeel-im im-ten-id-yettak I m-ten-yettekkes.

Avec action $=$ POSS2FS RELI IND2FS $=$ DIR3MP $=$ PROX $=S U J 3 M S-$ donner. AORI RELI IND2FS=DIR3MP=PROX= SUJ3MS-reprendre. AORI

Zoubida éclata en sanglot sans riposter, car cela n'aurait fait que confirmer les propos de sa belle-sœur. Alors que quelques voisines la consolaient, elle implora Dieu en ces termes : «Mon Dieu, c'est Toi que j'implore, Tu m'as donné des garçons et Tu les as repris, c'est Ton bien et Tu as le droit de vie et de mort sur eux, mais si ma situation te touche dans Ton point d'honneur (litt. « Si le nnif arrive vers Toi »), pour ces mots, donne-moi un garçon, à moi aussi ».

La réplique « regardez-la, la jalouse » ferme toute possibilité à Zoubida de se défendre par le jeu de l'interaction rituelle qui permet à la fois la reconstruction positive de soi et la possibilité de réintégrer la vie sociale. La référence à la perte de ses garçons sera très redondante, ainsi que nous le verrons dans l'exemple suivant.

Les disputes entre ces deux belles-sœurs ont très souvent pour élément déclencheur leurs enfants. C'est ainsi que Zoubida s'est vue affublée d'une tamzayert [moquerie méchante] pour avoir grondé les enfants de sa belle-sœur Ouardia : « vous êtes si turbulents que je me demande comment vous n'avez pas percé le ventre de votre mère ? ». Ce à quoi rétorqua la belle-sœur : 
Pauvre de toi.

A lhif-im.

$\mathrm{O}$ misère $=\mathrm{POSS} 2 \mathrm{FS}$

C'est toi dont le ventre est percé par les opérations.

D kemm i umi yefla ucebbuḍ sparasyuwat.

PP toi à qui SUJ3MS-être troué. PRET ventre. EA avec opérations.

L'expression « percer le ventre » est employée à l'adresse des enfants comme une hyperbole. Elle sert à exprimer de manière exagérée l'état d'excitation bruyante dans laquelle se trouvaient les enfants au point qu'ils auraient pu se frayer un passage en faisant une ouverture lorsqu' ils étaient dans le ventre de leur mère.

Ressentant les propos de Zoubida comme une agression, Ouardia répliquait là en se saisissant d'événements douloureux de sa vie, ses fausses-couches. Elle avait subi des césariennes, mais ses deux garçons n'avaient pas survécu. La formulation « le ventre percé » est par métaphore comme un récipient troué (ici par les césariennes) qui laisse filer tout ce qui aurait dû être retenu, à savoir des garçons viables.

La violence des propos de Ouardia prononcés à l'égard de Zoubida tire sa légitimité de sa supériorité sur cette dernière en tant que mère de garçons. L'offense de cette injure est si intense que sa vengeance nécessite une intervention divine. En guise de réponse, Zoubida fit le serment suivant :

Tu t'es moquée de moi pour mes opérations, alors je jure par Dieu que toi aussi tu en subiras.

Teggid-iyi tamcayert af parasyuwat gulley ar ad tt-txedmed ula d kem. SUJ2S-faire. PRET=IND1S moquerie sur opérations SUJ1S-jurer. PRET que POT DIR3FS=SUJ2S=faire. AOR même PP toi

Puis elle me confia s'être adressée à Dieu sur le balcon, les mains tendues vers le ciel :

Si j'ai droit à une malédiction alors qu'elle subisse aussi une opération.

Ma yella ttalasey ddnub ad texdem parasyu ula d nettat.

Si SUJ3MS-être. PRÊT SUJ1S-devoir. PRÊT péché POT SUJ3FS-faire.

AOR opération aussi PP elle

Ce fût le cas, Ouardia subira une hystérectomie du fait d'un fibrome utérin. « Si j'ai subi des césariennes c'était pour donner naissance à des garçons, même si Dieu me les a repris, me dit Zoubida. Quant à elle, elle l'a subie à cause d'une maladie vile qui lui a causé la stérilité ! ». La malédiction provoque ainsi un 
retournement du stigmate par le biais de l'énoncé, ce qui d'un point de vue social désamorce l'image humiliante infligée.

\section{Conclusion}

Tandis que l'injure met à l'épreuve le statut de l'individu dans une logique ritualisée dans laquelle la riposte est nécessaire pour rétablir l'équilibre de l'honneur, la malédiction n'attend pas de réplique et n'établit pas d'échange « elle agit avec force par la simple action de dire »(Giordano, 2014, p. 1). Elle est le plus souvent prononcée en l'absence du destinataire et se présente comme « une sentence » qui sera appliquée par une instance divine. L'efficacité de la malédiction est fondée sur la croyance en la capacité que peut avoir un individu d'enfermer par le biais d'un énoncé sa victime dans un sort qu'elle subit de manière immédiate ou différée. La plus redoutée est certainement celle proférée par des personnes détentrices d'un pouvoir légitime. Toutefois, lorsqu'elle est émise entre des personnes de statuts identiques, j’entends par là des femmes, la malédiction tire sa puissance des actes répréhensibles dont elles seraient victimes et qui s'avère également être d'une redoutables efficacité.

\section{Bibliographie}

Abrous Dahbia, 2010, « Malédiction » in Encyclopédie berbère, n 30, Édisud, Aix-en-Provence, p. 4540-4542.

Agamben Giorgio, 2009, Le Sacrement du langage : archéologie du sacrement. Homo sacer, Tome 2 et 3, Vrin, Paris.

Amri-Salameh Nelly, 2006, « La malédiction du saint. Du'â’ et situations de conflit dans l'Ifrîqiyya médiévale. Essai de typologie » in Hénia A. (Dir.), Etre notable au Maghreb. Dynamique des configurations notabiliaires, Institut de recherche sur le Maghreb contemporain, Rabat, p. 69-88.

Aubaile-Sallenave Françoise, 1988, « Le sel d'alliance » in Journal d'agriculture traditionnelle et de botanique appliquée, n 35, p. 303-323.

Aubaile-Sallenave Françoise, 1997, « Le monde traditionnel des odeurs et des saveurs chez le petit enfant maghrébin » in Enfance, n ${ }^{\circ}$, p. 186-208. 
Bourdieu Pierre, 2000, Esquisse d'une théorie de la pratique : précédé de trois études d'ethnologie kabyle, Éditions du Seuil, Paris, 448 p.

Chaker Salem, 1997, « Euphémisme » in Encyclopédie berbère, $\mathrm{n}^{\circ} 18$, Édisud, Aix-en-Provence, p. 2706-2707, http://journals.openedition.org/ encyclopedieberbere/2009.

Dallet Jean-Marie, 1982, Dictionnaire kabyle-français : parler des At Mangellat, Algérie, Société d'études linguistiques et anthropologiques de France (Coll. «Etudes ethno-linguistiques Maghreb-Sahara »), Paris, 259 p.

FÉDRY Jacques, 2010, Anthropologie de la parole en Afrique, Karthala, Paris, 357 p.

GÉlard Marie-Luce, 2004a, « Khebbach le père. Récit de fondation d'une tribu berbérophone du sud-est marocain $\gg$ in Revue Iris, $\mathrm{n}^{\circ} 27$, p. 399-412.

GÉLARD Marie-Luce, 2004b, « Modifications et ajustements événementiels des parentés électives dans le sud-est marocain » in Awal. Cahiers d'études berbères, $\mathrm{n}^{\circ} 29$, p. 51-64.

GÉLARD Marie-Luce, 2016, Les Pouvoirs du lait : coallaitement collectif et parenté en contexte saharien : Aït Khebbach, sud-est marocain, Éditions Pétra (Coll. «Terrains et théories anthropologiques »), Paris, $270 \mathrm{p}$.

Giordano Manuela, 2014, «Injure, honneur et vengeance en Grèce ancienne » in Cabiers « Mondes anciens », $\mathrm{n}^{\circ}$ 5, http://journals.openedition.org/ mondesanciens/ 1238

Goffman Erving, 1974, Les Rites d'interaction, Les Éditions de Minuit, Paris, $240 \mathrm{p}$.

Goffman Erving, 2005, Stigmate : les usages sociaux des handicaps, Les Éditions de Minuit, Paris, 176 p.

Gokalp Altan, 2004, « Les Ilenti ou maudire son prochain en turc » in Revue des Mondes musulmans et de la Méditerranée, no 103-104, p. 223-249, http:// journals.openedition.org/remmm/1207 


\section{CAHIERS DE LITTÉRATURE ORALE}

116 Éclats de paroles $-n^{\circ} 85$

Haddadou Mohand-Akli, 2014, Dictionnaire de tamazight - Parlers de Kabylie, Berti Éditions, Alger, 1058 p.

Khichane Samia, 2018, Ethnographie des conflits domestiques en Kabylie : injures, commérages, malédictions. Thèse de doctorat en anthropologie, Sorbonne Nouvelle-Paris 3.

Khichane Samia, à paraître, « Enquêter sur l'injure féminine en Kabylie : avantages et difficultés d'une ethnographie du proche » in LeBLIC I. \& Souag L (dir.), Du terrain à la théorie. Les 40 ans du Lacito, Publications du Lacito, (coll. Actes de colloque 1), Vuillejuif.

Lacoste-Dujardin Camille, 1970, Le Conte kabyle : étude ethnologique, François Maspero, Paris, 487 p.

Lacoste-Dujardin Camille, 2003, Voyage d'Idir et Djya en Kabylie. Initiation à la culture Kabyle, L'Harmattan, Paris, 134 p.

LaOust-Chantréaux Germaine, 1990, Kabylie côtéfermmes. La sociétéféminine à Aït Hichem, 1937-1939, Édisud, Aix-en-Provence, 304 p.

LARguèche Évelyne, 1998, Injure et sexualité : le corps du délit, Presses universitaires de France, Paris, 176 p.

Miliani Hadj, 1986, « Conduites et imaginaires sociaux du monde féminin en Algérie à travers l'insulte » in Les discours étrangers, OPU, Alger, p. 85-127.

Miliani Hadj, 2008, « Des “mauvaises” manières de parler des mères aux vannes des jeunes » in TAUZIN A. (dir.), Insultes, injures et vannes en France et au Maghreb, Karthala, Paris, p. 61-76.

Stillman Norman Arthur, 2008, « Invectives et malédictions affectueuses et injurieuses en judéo-arabe marocain » in TAUZIN A. (Dir.), Injures, insultes et vannes - En France et au Maghreb, Karthala, Paris, p. 11-32.

TAIne-Cheikh Catherine, 2004, « De l'injure en pays maure ou "qui ne loue pas critique” » in Revue des Mondes musulmans et de la Méditerranée, $\mathrm{n}^{\circ}$ 103-104, p. 103-126. 
Yacine Tassadit, 1995, Piège ou le combat d'une femme algérienne : essai d'anthropologie de la souffrance, Publisud, Paris, 213 p.

YACINE Tassadit, 2001, Chacal ou la ruse des dominés : aux origines du malaise culturel des intellectuels algériens, La Découverte, Paris, 287 p.

ZAGNOLINello, 1991, «Figures et logique du serment » in VERDIER RAYMOND R. (dir.), Le Serment : théories et devenir, Tome 2, Éditions CNRS, Paris, p. 137-199.

Zerdoumi Nefissa, 1970, Enfant d'hier: l'éducation de l'enfant en milieu traditionnel algérien, François Maspero, Paris, 301 p.

Résumé : En Kabylie, la malédiction est une pratique en usage avant tout chez les femmes. Elle intervient notamment dans leurs rapports à leurs enfants, comme pratique éducative, mais également dans le cadre des querelles et des disputes qui les opposent entre elles dans l'univers domestique ou (très rarement) aux hommes. Le recours à des pratiques détournées telle que la malédiction est pour elles, en tant que groupe dominé, l'unique moyen de se défendre des représailles que leur inflige la société sans contrevenir à la bienséance.

Mots-clefs : Kabylie, malédiction, femmes.

\section{The Curse : Power of Weaks or Redoutable Power?}

Abstract: In Kabylia, the curse is a practice in used over all by women, it intervenes in their relationship with their children, as an educational practice, but also in the context of quarrels and disputes that oppose them in the domestic universe or (very rarely) to men. As a dominated group, the use of diverted practices such as the curse is the only means for them to defend themselves against reprisals inflicted on them by society without contravening propriety.

Keywords: Kabylia, curse, women.

Samia Khichane est docteur en anthropologie de l'université Sorbonne Nouvelle-Paris 3, chercheure associée au LACITO (Langues et civilisations à traditions orales, CNRS), ses recherches portent sur les injures chez les femmes kabyles. Sa thèse de doctorat s'intitule : Ethnographie des conflits domestiques en Kabylie: injures, commérages, malédictions. 